\title{
Chacun pour soi(e) ? Lobbying, stratégies économiques et commerce international dans le secteur de la soie aux Pays-Bas espagnols (1618)
}

\author{
Nicolas Simon
}

\begin{abstract}
Nicolas Simon : Diplômé en histoire de l’UCL (2011), Nicolas Simon a également réalisé un master complémentaire en sociologie-anthropologie (UCL, 2012). Il est actuellement Aspirant F.R.S-FNRS. L'axe principal de ses recherches s'insère dans les champs de l'histoire du droit, en particulier celle de la législation, et de l'histoire interne des institutions. Sa thèse de doctorat porte sur l'étude du processus décisionnel au niveau législatif dans les Pays-Bas espagnols (fin XVIe-début XVIIe siècle).
\end{abstract}

DOI: $10.25518 / 1370-2262.327$

\section{Résumé :}

En mars 1618 fut promulguée, dans les Pays-Bas espagnols, une ordonnance interdisant l'importation de soies issues de territoires étrangers, c'est-à-dire non soumis à la juridiction des archiducs Albert et Isabelle. Cette ordonnance n'est pas la première du genre puisqu'un précédent acte avait déjà été promulgué en 1606 à la suite d'une requête envoyée par la ville d'Anvers et le métier des merciers de cette même ville. Plus globalement, l'acte de 1618 s'intègre dans la logique des ordonnances dites somptuaires visant à réglementer les habillements jugés trop luxueux. À la suite de l'ordonnance de mars 1618, une campagne de lobbying est menée par deux parties opposées. D’un côté se trouvent les marchands, adversaires de l'interdiction d'importer des soies étrangères. Aux antipodes de cette position se trouvent les préparateurs de la soie de cités flamandes et brabançonnes pour qui l'interdiction signifie la possibilité d'écouler plus facilement le fruit de leur labeur. La présente contribution se propose d'analyser le contexte particulier dans lequel fut prise cette ordonnance de même que l'argumentation et les stratégies narratives utilisées par les préparateurs de soie dans leur requête.

Mots-clés : commerce international - Trêve des Douze Ans - lobbying - archiducs Albert et Isabelle

\section{Introduction}

«Touttes lesquelles raisons plaira a voz Altezes Serennissimmes prendre en bonne consideration et que par ainsi leur bon plaisir soit de faire continuer et renouveller ledict tant equitable et utile placcart par touttes leurs villes et commander expressement qu'il soit inviolablement maintenu et observé au bien consolation de leurs humbles subiects parmy tant de leurs villes » 1 . Par cette phrase se termine la longue requête (30 articles) adressée aux archiducs Albert et Isabelle et envoyée, en 1618, par les préparateurs de soie de plusieurs villes des Pays-Bas espagnols dont Anvers, Bruges, Malines, Lierre et Termonde. Cette requête mérite que l'on s'y attarde dans la mesure où elle émane de villes importantes pour l’histoire économique et politique de cette région. En réalité, ce document s'apparente à une clé donnant accès à plusieurs univers. Premièrement, la requête est rédigée au cours de la Trêve de Douze Ans (1609-1621), c'est-à-dire 
Chacun pour soi(e) ? Lobbying, stratégies économiques et commerce internation...

cet accord temporaire entre les Pays-Bas catholiques/espagnols et les Provinces-Unies calvinistes durant le règne des archiducs Albert et Isabelle. Ces années ont été abondamment étudiées, notamment à l'aune des réalités juridiques et économiques que la trêve impliqua pour les provinces catholiques2. Deuxièmement, il convient de souligner l'identité des requérants. Divers éléments doivent sans doute retenir ici l'attention. Le type d'activité (la préparation de la soie) nous renvoie au commerce d'un textile coûteux, luxueux et dont l'usage fit l'objet de réglementations relevant de la législation dite somptuaire $\underline{3}$. La présentation d'une seule requête par des métiers issus tant du comté de Flandre que du duché de Brabant est assurément un autre élément à ne pas négliger. Troisièmement, nous pouvons le type de document qui fera l'objet de notre attention. Soumettre une requête aux Archiducs, qui plus est pour demander le maintien d'une ordonnance que certains marchands voulaient vraisemblablement voir abroger, est symptomatique des modalités propres à la communication entre gouvernants et gouvernés.

Avant de s'attarder sur ces différents points d'analyse, il faut, en premier lieu, poser les bases du débat. La requête des métiers de la soie permet de nous insérer dans un courant historiographique qui, sans être totalement neuf, n'a pas bénéficié d'une grande attention de la part des chercheurs jusqu'il y a peu. En effet, l'étude des institutions politiques à l'époque moderne pré-industrielle tend davantage aujourd'hui à intégrer une réalité fondamentale : l'État n'est pas un artefact, une chose imposée sans réflexion par les gouvernants aux sujets4. S'il faut sans doute reconnaître qu'une impulsion peut venir « d'en haut » (i.e. les souverains et leur appareil institutionnel) $\underline{5}$, il ne faut pas sous-estimer les dynamiques produites «par le bas » (i.e. les sujets, les gouvernés au sens large). Une variété de groupes sociaux - les métiers en font naturellement partie - ont pu recourir aux moyens juridiques et matériels en leur possession afin d'atteindre leurs buts. En ce sens, ces groupes sociaux ont pu participer à la construction d'ensembles institutionnels et politiques à l'époque moderne․ Ainsi, le dépôt d'une requête n'est pas un geste gratuit, mais bien un moyen à privilégier si un sujet, une ville, un groupe de villes ou de métiers souhaitaient être entendus par les autorités politiquesz. Jelle Haemers a récemment rappelé que les recherches en histoire urbaine se sont longtemps satisfaites de retenir la révolte comme modalité privilégiée des villes pour exprimer leurs revendications auprès du prince. Il est toutefois patent que d'autres méthodes ont pu être employées. La rédaction d'écrits destinés à être diffusés au sein de la ville, le recours à la communication orale (chansons, poèmes) afin de propager une rumeur ou encore l'usage de rituels ou de cérémonies non violentes (sonner les cloches) sont d'autres indices de la pluralité des moyens d'expression à disposition des sujets. Il faut donc encore ajouter à cette liste les requêtes $\underline{8}$. Éventuellement orales $\underline{9}$, les requêtes pouvaient être envoyées aux autorités sous forme écrite en mobilisant, par ce biais, des stratégies narratives propres à assurer une prise en compte des revendications émises 10 . L'historiographie récente tend également à mettre en exergue qu'une ordonnance princière faisant mention d’une requête reprend généralement dans le préambule de l'acte final les mots exacts de ladite requête11. Pour autant, d'autres sources d'inspiration peuvent se retrouver dans la mouture finale du prescrit légal12. Cette dernière remarque est d'ores et déjà capitale, nous semble-t-il, car elle interroge sérieusement la vision d'une législation imposée unilatéralement par les plus hautes strates du pouvoir politique.

Cette capacité à produire des écrits structurés destinés à mobiliser le prince et ses institutions invite le chercheur à investiguer plusieurs fronts. Pour la période qui nous occupe ici - début $\mathrm{du} \mathrm{XVII}^{\mathrm{e}}$ siècle - émerge la question des conditions d'existence d'une opinion publique capable de structurer et de diffuser une idée et/ou une pensée cohérente. Des critiques ont toutefois été formulées à l'encontre des thèses sur l'Öffentlichkeit telles que développées par Jürgen Habermas. 
D'aucuns soulignent que la société des temps modernes dispose d'un «système multimédia » où le support écrit n'est pas le seul moyen utilisé pour diffuser ou transmettre une information 13 . Toutefois, il est vrai qu'avant le $\mathrm{XVII}^{\mathrm{e}}$ siècle, il n'existe pas de périodiques ou de quotidiens permettant d'être informé de manière large à propos de matières politiques ou économiques. La collecte d'informations était avant tout l'apanage d'individus informés sur la base de réseaux sociaux et d'échanges14. Ceux-ci pouvaient ainsi aider à forger un avis, une opinion et, partant, conduire à la production de sens. Il ne faut sans doute pas perdre de vue cette donnée si l'on souhaite procéder plus avant dans l'analyse des tenants et aboutissants de la rédaction d'une requête par une pluralité d'agents sociaux.

Les chercheurs ayant étudié le processus législatif dans les Pays-Bas bourguignons au $\mathrm{XV}^{\mathrm{e}}$ siècle sont généralement prompts à souligner le nombre relativement faible de requêtes ou de documents préparatoires à disposition des historiens 15 . Ceci peut s'expliquer par la nature même de la requête qui n'est pas systématiquement conservée après qu'une décision soit prise. À l'inverse, le chercheur étudiant la même thématique aux $\mathrm{XVI}^{\mathrm{e}}$ et $\mathrm{XVII}^{\mathrm{e}}$ siècles sera confronté à une certaine prodigalité des sources16. C'est dans cette veine qu'il nous a paru important d'étudier le document produit par les métiers de la soie en 1618. Qui plus est, mettre en lumière l'importance que revêtaient les requêtes et pétitions dans le secteur économique relève presque du truisme 17.

Dans cette contribution, on tâchera, dans un premier temps, de présenter les contextes politique et économique dans lesquels prit place cette requête des métiers de la soie. La seconde partie de l'exposé se propose d'approfondir l'analyse : quelle stratégie narrative adoptent les métiers ? Qu'apprend-on de la situation socio-économique des Pays-Bas espagnols au XVII ${ }^{\mathrm{e}}$ siècle ? Pouvonsnous replacer le problème traité dans la plus longue durée ? Au-delà de cette approche casuistique, que pouvons-nous tirer comme enseignement par rapport à l'étude du processus décisionnel sous les Archiducs?

\section{Économie, politique et réseaux internationaux}

L'année où la requête des métiers de la soie parvint au gouvernement central des Archiducs, les principautés des Pays-Bas espagnols connaissent une période particulière de leur histoire politique. Depuis presque dix ans est alors en vigueur la trêve signée en avril 1609, à Anvers, avec les Provinces-Unies de Maurice de Nassau, stadhouder de Hollande18. La situation de paix provisoire prit fin en 1621 faute d'accord pour la prolonger19. Cette dizaine d'années permit notamment le développement économique des Provinces-Unies et obligea la Monarchie espagnole à reconsidérer ses relations avec ces territoires, vus par Madrid comme rebelles depuis l'acte de déchéance prononcé à l'encontre de Philippe II en 1581. Les monarques hispaniques furent en réalité incapables de juguler l'afflux de biens manufacturés en provenance du nord de l'Europe et singulièrement ceux de la Hollande. La production textile castillane connut ainsi une contraction relativement forte en raison du déferlement des produits en provenance des Provinces-Unies20. En 1616, le président du Consejo de Hacienda souligna même que la situation économique de la monarchie depuis la trêve était pire que durant la période de guerre qui avait précédé21. En effet, la Trêve de Douze Ans avait amené la Monarchie espagnole à lever l'embargo sur les produits issus des provinces rebelles tout en adoptant certains accommodements fiscaux $\underline{22}$. Les premières années $\mathrm{du} \mathrm{XVII}^{\mathrm{e}}$ siècle marquèrent donc une accélération des activités économiques des Provinces-Unies sous l'impulsion notamment de la Verenigde Oost-Indische Compagnie (VOC) créée en 160223. Fernand Braudel a jadis popularisé l'idée d'une arrivée massive des Provinces-Unies dans les 
Chacun pour soi(e) ? Lobbying, stratégies économiques et commerce internation...

échanges commerciaux méditerranéens au cours des années 159024. S'il est vrai que les ennemis jurés du roi d'Espagne ainsi que les Anglais prirent progressivement le contrôle du commerce entre l'Europe du Nord et la Méditerranée, d'aucuns tendent toutefois à préciser que d'autres agents économiques (Grecs et Ottomans) restèrent des acteurs importants du commerce méditerranéen25. En outre, des contacts économiques existaient entre l'Europe du Nord et la Méditerranée bien avant la décennie 1590-160026. Ainsi, les marchands issus des Pays-Bas ou des Provinces-Unies n'arrivent pas soudainement à Venise, Gênes ou Ancône à la fin du XVI ${ }^{\mathrm{e}}$ siècle27. Ceci n'est pas anodin puisque l'approvisionnement des Pays-Bas en soie passait par la péninsule italienne...

Afin de mieux comprendre les éléments repris dans la requête des métiers de la soie, il nous faut également rappeler brièvement la situation économique d'Anvers au sein du commerce international. Dès le XIV ${ }^{\mathrm{e}}$ siècle, les principautés des anciens Pays-Bas entretenaient des relations commerciales avec la Méditerranée par l'intermédiaire de voyages effectués par les navires vénitiens. Des convois annuels en provenance de la cité du Doge apportaient à Londres et Bruges des épices, du coton ou encore de la soie en provenance du Levant. Les navires italiens repartaient chargés de textiles anglais et de produits achetés aux marchands hanséatiques postés à Bruges $\underline{28}$. La fin $d u X V^{e}$ siècle fut ensuite marquée par la montée en puissance d'Anvers comme place marchande internationale29. Le premier tiers du XVI siècle coïncide avec l'affirmation de la cité brabançonne dans le jeu des échanges économiques européens ; elle est d'ailleurs la première ville au nord des Alpes à compter plus de 100000 habitants $\underline{30}$. L'importance du commerce international et la présence de marchands étrangers étaient d'ailleurs, selon F. Guichardin, la cause de la prospérité anversoise; la ville s'apparentait à un microcosme du monde $\underline{31}$. La seconde moitié du $\mathrm{XVI}^{\mathrm{e}}$ siècle voit toutefois l'étoile d'Anvers pâlir en raison des troubles internes aux XVII Provinces. En 1585, la ville est certes reprise par Alexandre Farnèse, gouverneur général des Pays-Bas au nom de Philippe II, mais l'accès à la mer du Nord était dorénavant impossible en raison du blocage effectué par les provinces de l’Union d'Utrecht32.

Si le commerce entre Anvers et Venise fut si important au cours du XVI ${ }^{\mathrm{e}}$ siècle, c'est notamment en raison de la position géographique de la cité italienne. La majeure partie des produits en partance d'Anvers n'avait pas Venise pour destination finale mais bien le Levant33. À Venise même, la forte présence de marchands issus des Pays-Bas n'est pas à négliger. L'influence toujours plus grande de ces marchands non vénitiens sur la politique de la cité conduisit même les élites locales à interpeller le Doge au début du XVII ${ }^{\mathrm{e}}$ siècle $\underline{34}$. De son côté, Anvers avait pu attirer un nombre croissant de commerçants étrangers à l'intérieur de ses murs. On fournit aux marchands anglais (Adventurers), en 1550, le Hof van Liere construit par un ancien bourgmestre de la ville (Aert/ Arnold van Liere) et qui avait été racheté par la ville après son décès. La Hanse disposait également d'un établissement (Oosterlinghenhuis) depuis 156835. Il est significatif de souligner qu'Anvers était alors principalement un hub européen de redistribution par voies terrestres $\underline{36}$. En s'attardant davantage sur le commerce de la soie, on observe qu'Anvers devient un centre important de transit pour le marché continental dès le milieu du $\mathrm{XVI}^{\mathrm{e}}$ siècle. Les soies provenaient majoritairement de la péninsule italienne même si une industrie propre aux Pays-Bas s'était développée 37 . Les soies étaient ensuite exportées vers Londres, la France ou en direction de la Baltique. Le dernier tiers du $\mathrm{XVI}^{\mathrm{e}}$ siècle vit ensuite l'explosion de la passementerie 38 sans doute aidée par la présence, à Anvers, de Génois ou de Vénitiens spécialisés dans ce domaine $\underline{39}$. En 1584, on comptait environ 4000 travailleurs dans ce secteur à Anvers pour une population globale de ca. 80000 âmes. Un autre indicateur de l'importance du marché de la soie est la croissance du nombre de marchands 
ou de revendeurs entre 1534 et 1597 puisque l'on observe l'arrivée de 56 individus $\underline{40}$. La requête adressée aux Archiducs en 1618 indique que « dedens la ville d'Anvers seulle en sont bien environ cinq ou six milles personnes propres a travailler és soyes comme par plusieurs foys on at monstré a messieurs les commissaires » du gouvernement central. On signale dans le même paragraphe que « vray est que Anvers ne sont qu'environ cincquante maistre preparateurs de soyes mais est a noter que chacun maistre en a bien cent ouvriers, l'ung parmy l'aultre, estant assez ladicte manufacture augmentée és villes de Vallenchiennes, Gand et Lille (...) »1ㅗ. À la même époque (1614), les marchands flamands $\underline{42}$ en poste à Venise faisaient pression auprès des autorités urbaines pour disposer d'un privilège sur les soies crues importées de Syrie. Jusqu'au début des années 1630, Venise reçut aussi quantité de requêtes de la part de ces marchands demandant la suppression de taxes douanières ou la permission d'emprunter d'autres routes terrestres que celles imposées par la Sérénissime $\underline{43}$.

Comme on le voit, tant les métiers que les marchands pouvaient exercer une certaine pression sur les autorités politiques dans le but de servir leurs propres intérêts. Ces deux groupes sont régulièrement en concurrence puisque production (métiers) et commerce (marchands) ne sont pas encore étroitement imbriqués. En amont de la commercialisation, il est patent de constater que les métiers anversois vont généralement tendre à augmenter les contrôles ex ante afin d'objectiver la qualité des produits. Ce n'est pas anodin compte tenu des arguments émis par les métiers de la soie en 1618 (cf. infra). Quoi qu'il en soit, la qualité supérieure des produits manufacturés autorisait à espérer une bonne réception de ceux-ci au moment de la vente et/ou de l'exportation. In fine, l'offre et la demande n'étaient pas les éléments premiers pris en compte par les stratégies corporatives. Il s'agissait avant tout de créer une image de marque qui passait inévitablement par la bonne qualité du produit. Étant donné que le marché anversois était principalement orienté vers les biens de luxe, on peut comprendre que l'attention se soit portée sur cet élément-là. Il ne s'agissait toutefois pas non plus d'unifier production et distribution puisque pareille imbrication aurait estompé l'idée de la qualité comme élément déterminant à retenir44.

\section{La stratégie des métiers de la soie : favoriser la production locale}

La requête envoyée par les métiers de la soie en 1618 faisait écho à l'ordonnance publiée le 3 mars de la même année interdisant l'importation de «soyes crues, ouvrées ou teintes, soit en bales, en groz ou pour vendre en menu, venus d'aulcuns pays ou provinces » qui ne relevaient pas de la juridiction des Archiducs $\underline{45}$. L'acte promulgué mettait en lumière une relative perte de vitesse des travailleurs des soieries dans les Pays-Bas habitués à travailler avec des « soyes crues venant de Sorye [Syrie] et d'aulcuns quartiers de Levant ». En effet, la raison de la faible croissance de ce secteur économique s'expliquait par l'arrivée sur le marché des Pays-Bas de produits venant de France, du Saint-Empire et de la principauté ecclésiastique de Liège. L'ordonnance visait donc prioritairement à protéger les préparateurs de la soie des Pays-Bas - principalement de Flandre et du Brabant - de la concurrence étrangère느. La requête des métiers s'y réfère d'ailleurs abondamment. L'ordonnance de mars 1618 renouvelait aussi des dispositions promulguées en 1606. Cette année-là, l'ordonnance du 27 septembre tentait de légiférer à propos de la teinture des soies. Plutôt que de nettoyer correctement la soie brute avant de la teindre, certains n'hésitaient apparemment pas à recourir à des « gresses et aultres substances indues et corrosives ». Dès lors, à la pesée lors de la vente, les pièces de soie ainsi teintes pesaient plus lourd et garantissaient aux revendeurs certains bénéfices frauduleux. Qui plus est, ces produits manufacturés de la sorte étaient de moins bonne qualité et risquaient de se détériorer plus rapidement. L'acte de 1606 
Chacun pour soi(e) ? Lobbying, stratégies économiques et commerce internation...

interdisait de faire venir ou d'acheter dans les Pays-Bas « aulcune soye taincte ou cru ou chargée ny aulcuns ouvraiges faictz d'icelle (...) exceptéz seulement les velours d'Italie » $\underline{4}$. En réalité, des pressions s'étaient exercées sur le gouvernement central dès 1605 par l'intermédiaire d'une requête envoyée par la ville d'Anvers. Les autorités de la ville associées aux merciers informaient le Conseil privé que des fraudes étaient commises concernant la teinture de la soie de sorte que

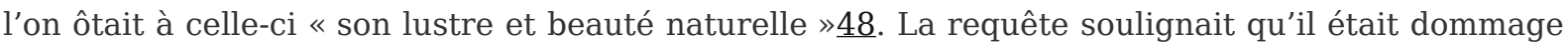
que les autorités des Pays-Bas n'avaient pas encore pris de disposition à cet égard alors que les «rebelles et ennemys » des Provinces-Unies l'avaient fait $\underline{49}$. Dans une apostille datée du 5 septembre 1605, le Conseil privé ordonnait ainsi au Conseil de Brabant de fournir son avis après avoir entendu «quelques principaulx marchans d'Anvers ». En outre, il convenait aussi d'envoyer un double de cette requête "aux lieutenants de Lille et Tournay respectivement pour aussy oyr quelques bons marchans desdites villes et donner leur advis » $\underline{00}$. En 1618, le gouvernement central profitait ainsi d'une nouvelle ordonnance pour rappeler que ces dispositions de 1606 n'étaient pas caduques. Néanmoins, l'acte de 1618 précisait que ne sont pas comprises dans les soies interdites celles d'Italie et d'Espagne à condition de s'être acquitté du paiement des droits « pour ce accoustuméz » $\underline{1}$. En 1606, plusieurs magistrats urbains avaient visiblement essayé d'endiguer les fraudes liées à la teinture de la soie (cf. supra). Toutefois, ces tentatives ne produisirent par les effets attendus de sorte qu'on en appela au gouvernement central : «(...) aulcuns des magistratz de noz bonnes villes ayent voulu remedier et la deffendre par particuliers statutz et ordonnances, ce neantmoins comme elles ne sont esté generales » les désordres continuèrent $\underline{52}$. Cette manière de procéder ne semble pas isolée si bien que l'échelon central paraît être celui mobilisé lorsqu'une solution locale ne pouvait être trouvée.

Signalons encore que le début du XVII ${ }^{\mathrm{e}}$ siècle est marqué par la tentative d'un échevin du Franc de Bruges, Thomas Gramaye, de développer la culture de mûriers dans les Pays-Bas $\underline{3}$. Par ce biais, Gramaye espérait limiter la fuite des capitaux des Pays-Bas vers d'autres contrées pour l'achat de soies brutes ou manufacturées. Il entendait amener dans les principautés des Archiducs la culture du fil de soie à l'instar de la France à la même époque $\underline{44}$. L'échevin déposa une requête auprès des Archiducs afin de pouvoir procéder à l'achat et à la plantation de 400000 plants de mûriers pour ensuite les «faire planter en aucunes des principales provinces de pardeca » $\underline{5}$. Pour autant il ne semble pas que Gramaye ait pu atteindre quelque résultat probant $\underline{56}$.

Il faut rappeler qu'au cours du $\mathrm{XVI}^{\mathrm{e}}$ siècle, la soie avait fait l'objet d'attention de la part du gouvernement central par l'intermédiaire de textes relevant de la législation somptuaire, soit ces

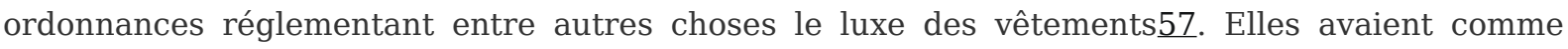
principal objectif de rappeler à chacun sa position dans la hiérarchie sociale en interdisant toute transgression 58. En 1531, on relève plusieurs articles réglementant les vêtements et habits sur les quarante-cinq que compte l'ordonnance du 7 octobre $\underline{99}$. Probablement inspiré d'une ordonnance publiée pour le Saint-Empire, l'acte de 1531 précédait les ordonnances du 30 janvier 1546 (n.st.) 60 et du 27 mai 155061. En mai 1594, le gouverneur général des Pays-Bas, l'archiduc Ernest, conseille encore à Philippe II de dresser une ordonnance somptuaire : "On me dit aussi, monseigneur, qu'il seroit expedient de faire quelques loix publicques que l'on appelle sumptuaires pour moderer et reprimer les grands despens et exces qui se commettent par les subiectz d'icy chacun en sa condition et qualité (...) »⒉ Le Conseil de Brabant suggère en septembre de la même année de " pourveoir aux sumptuositéz des habitz et despens excessifz de tous estatz comme aultrefois a esté faict par feu de trés haulte memoire l'empereur Charles le Quint » et de "faire renouveller les ordonnances et deffences des draps d'or, d'argent et de soye $\triangleq \underline{63}$. Comme on le voit, la question 
retenait donc l'attention des gouvernants et ce jusqu'à la personne du roi.

Si nous en venons maintenant à l'ordonnance de 1618, nous pouvons affirmer avec une certaine assurance que l'acte fut vraisemblablement conçu par le chancelier de Brabant, Pierre Peckius $\underline{64}$. Dans une lettre datée du 11 février 1618, celui-ci écrit à Louis Verreycken65, audiencier, qu'il lui a été demandé d'envoyer le " placcart sur le fait des soyes afin de le mettre en forme deue au plustost selon la resolution qui en a esté prinse par icelle son Alteze » $\underline{6}$. Pierre Peckius est davantage qu'un simple figurant puisqu'il apparaît comme «la » personne impliquée dans la rédaction de l'acte et semble avoir traité le dossier directement avec les Archiducs. Étant donné le leadership d'Anvers dans cette affaire, l'implication de Peckius n'est sans doute pas étonnante. Nous disposons ainsi de la minute de l'ordonnance en trois exemplaires, deux en français et un en thiois. De son côté, l'audiencier n'a pas traîné pour procéder à la mise au net du texte puisque l'ordonnance est promulguée une vingtaine de jours après la lettre de Peckius. La requête envoyée par les métiers fait vraisemblablement suite à une activité de lobbying de la part de marchands désireux de voir l'ordonnance annulée. Si la requête mentionne explicitement les métiers d'Anvers, Bruges, Termonde, Malines et Lierre, il faut supputer que d'autres villes furent également impliquées dans la manœuvre67. Toutefois, la prédominance d'Anvers est évidente. Tous les exemples servant à illustrer les arguments émis sont tirés du cas anversois. De manière générale, nous avons affaire à la tension mise en lumière plus haut concernant les rapports délicats entre les marchands, d'une part, et les métiers, d'autre part.

L'ordonnance de mars 1618 interdisait l'importation des soies liégeoises, françaises et allemandes. Ce facteur international est la clé de voûte de l'argumentation et le point central d'opposition entre les préparateurs de la soie et certains marchands qui ne chercheraient qu'à « disturber le bien commun pour leur singulier proffit ». La position des deux parties est, en réalité, très simple à résumer. Les marchands souhaitent l'abrogation de l'ordonnance dans la mesure où l'interdiction d'importer certaines soies risque de créer une pénurie dans ce secteur. Selon eux, la production locale des Pays-Bas ne suffirait pas à leur commerce. A contrario, les métiers soulignent l'importance des dispositions législatives. Ils mettent en exergue le tonus que pourra retrouver la production anversoise et, partant, celle de tous les Pays-Bas car le « sainct placcart [du 3 mars 1618] at causé grand bien et changement en ces pays et est par ce fort restaurée la manufacture desdictes soyes et une infinité de peuple, lequel auparavant ledict placcart parmy lesdictes villes estoient oisives et sans ouvraige (...) $» \underline{68}$.

Dès les premiers articles, les métiers stigmatisent les problèmes liés à l'importation de soies issues de France ou d'Angleterre. Plus globalement, il faut noter que leur argumentation fait la part belle à l'aspect international de leur activité. En cela, ces 30 articles illustrent bien l'expansion et les conditions du commerce (extra-)européen. Ainsi, si les marchands français ont longtemps acheté des "soyes crues a Venise, en Italie», provenant de Perse après un transit en Turquie, ces mêmes marchands préféraient dorénavant négocier directement avec les Turcs pour amener les soies à Marseille69. Cette situation implique que «ceulx des villes de Genevre, Lion et aultres villes et villetes, bourgs et villaiges circonvoisins s'en sont renforchéz a travailler desdites soyes plus qu'auparavant». En clair, les travailleurs des Pays-Bas entendent faire comprendre que l'expansion française se réalise au détriment de l'activité économique dans les territoires d'Albert et Isabelle. En Angleterre, rappelaient les métiers, Jacques $\mathrm{I}^{\mathrm{er}}$ a « chargé les soyes preparées en France avecq deux soulz esterlins la livre, de maniere qu'elles y sont bannies d'elles meismes $\gg \underline{70}$. De même, à Amsterdam, une taxe est perçue sur les soies françaises de sorte que ces produits 
Chacun pour soi(e) ? Lobbying, stratégies économiques et commerce internation...

n'entrent pas en concurrence avec la production locale71. Les métiers précisaient néanmoins qu'il est « notoir et manifeste pour tout le monde que les navigans d'Hollande et Zeelande amenent par les Indes Orientalles souventesfois des soyes crues du Royaume de China » qu'ils préparent ensuite à Amsterdam pour in fine les amener de manière illicite dans les Pays-Bas via Cologne 72 . Le message que veulent envoyer les métiers au gouvernement central est tout simplement que la suppression de l'ordonnance du 3 mars engendrerait une arrivée massive des soies de France ou de Liège et, partant, la création d'un libre marché de la soie se ferait en défaveur des travailleurs - entendez les préparateurs de soie - des Pays-Bas espagnols. Qui plus est, les métiers évoquaient l'interdiction qui frappait, en France, les soies issues des Pays-Bas고. Autoriser les produits français aurait dès lors été une aberration. En guise de menace, les métiers soulignaient que le roi de Danemark, Christian IV (r. 1588-1648), engageait des travailleurs, principalement en Hollande, afin de développer le commerce de la soie dans son royaume. Si d'aventure leurs revendications n'étaient pas entendues, les préparateurs des Pays-Bas seraient sérieusement « constraincts aussy d'y aller par ce que ledict placcart n'en est deuement observé (...) $\gg \underline{74}$. Le départ de travailleurs à l'étranger était d'ailleurs un problème récurrentz․

Pour asséner leurs arguments, les métiers soulignaient le nombre important des travailleurs dans ce secteur à Anvers (ca. 5000-6000 personnes). Dans le même temps, ils essaient de battre en brèche la portée des arguments avancés par les marchands en soulignant le nombre ridiculement bas de ceux-ci. Ils condamnent ainsi les «quelcque quinze ou vinte des plus riches marchands tenans bouticle de soyes és Anvers et une douzaine de plus riches marchans lapidaires » $\underline{76}$. Plus loin, on rappelait que "quelcques cincq ou six marchans d'Anvers » se sont plaints de ne pas trouver de soie travaillée dans la cité afin d'en vendre77. En outre, ces marchands sont accusés de revendre les soies françaises à un prix supérieur à celui auquel ils les ont achetées et en profiteraient pour accomplir une opération financière très rentableㄹ. En réalité, si les produits français étaient moins chers c'était, selon les métiers, en raison de leur moins bonne qualité79. Cette situation ne pose d'ailleurs pas seulement problème pour le commerce de la soie car tant les teinturiers que les passementiers ou le secteur de la tapisserie s'en trouveraient grandement amoindris si l'ordonnance était abrogée 80 .

Les arguments des métiers ont-ils porté leurs fruits ? À première vue on serait enclin à le penser. Ou du moins le gouvernement des Archiducs ne paraît pas avoir été prêt à transiger sur la question de la soie. Le 18 septembre 1618 un courrier fut envoyé au Conseil de Brabant et, pour information, à une dizaine d'autres institutions provinciales afin de rappeler l'application de l'ordonnance du mois de mars. Le courrier mettait en avant les problèmes causés par l'introduction, dans les PaysBas, de produits issus de territoires non sujets des Archiducs de même que le caractère labile des officiers dans la mise en application de l'ordonnance $\underline{81 .}$

\section{Conclusions}

Les autorités politiques, tant celles du niveau central que des échelons inférieurs, reconnaissaient l'importance des requêtes présentées par les sujets car elles renvoyaient à une pratique « légale » reconnue. Entendues comme un moyen pacifique de régler les conflits internes à une ou plusieurs principautés, les gouvernants étaient prompts à accorder du crédit aux propositions contenues dans ces requêtes et pétitions plutôt qu'être mis devant le fait accompli d'une révolte violentę2. La mise en commun des ressources afin de peser collectivement sur les décisions peut toutefois être un jeu à double tranchant. Si les villes importantes d'un point de vue économique se mobilisent 
dans les Pays-Bas en 1618, d'autres exemples européens montrent que certaines localités tâchent parfois de jouer cavalier seul83. Il est néanmoins flagrant que l'agenda législatif du gouvernement central dans les Pays-Bas était suivi de près par les marchands de sorte qu'ils n'hésitaient pas à entrer en conflit avec les métiers ou à effectuer un lobbying visant la modification de dispositions promulguées à l'instar du cas étudié dans cette contribution $\underline{84}$.

L'exemple de l'ordonnance de mars 1618 nous montre surtout l'obligation qu'ont les marchands et métiers de penser au-delà des murs d'Anvers. Les mesures prises par le gouvernement central des Archiducs s'assimilaient à une politique qu'on qualifierait aujourd'hui de protectionniste puisque destinée à favoriser les travailleurs locaux. Vingt ans avant cet acte, rappelons qu'une ordonnance de l'empereur Rodolphe II, frère de l'archiduc Albert, était promulguée dans les Pays-Bas à sa propre demande - elle avait été traduite de l'allemand pour l'occasion. Celle-ci interdisait la présence des marchands anglais de la compagnie des Adventurers et faisait écho aux multiples tracas juridiques que connaissaient les villes de la Hanse en Angleterre85. Ici comme là, c'est à l'échelle du continent que les politiques s'envisagent, si ce n'est à celle du monde. Comme l'expriment les métiers de la soie dans leur requête, le« monde est grand » et le temps où les échanges se cantonnaient à une ville et son hinterland est désormais révolu⒍ La relative nouveauté est peut-être l'intervention de marchands étrangers dans le processus décisionnel alors qu'ils ne maîtrisaient pas toujours les habitudes locales $\underline{87}$.

L'analyse de cette requête des métiers de la soie soulève différentes pistes de réflexion. Premièrement, on est en droit de s'interroger sur le chemin effectivement parcouru par la requête pour arriver entre les mains du gouvernement central : quel(s) intermédiaire(s) ? Quel lobbying pour s'assurer une prise en compte des revendications ? Au début du règne des Archiducs plusieurs individus furent officiellement reconnus comme agents auprès du Conseil privé et, partant, autorisés à présenter les requêtes à destination de cette institution centrale. Si l'on dispose de quelques informations à propos de ces agents pour le XVIII ${ }^{\mathrm{e}}$ siècle, tout (ou presque) reste à faire pour le XVII siècleㄹ. De cette remarque en découle une autre concernant le rôle du gouvernement central dans l'élaboration de la législation. Si l'autorité et la légitimité des Archiducs sont recherchées car un texte pris en leurs noms disposait d'une aura supérieure, il faut reconnaître que le gouvernement central s'appuyait presque entièrement sur les documents qui lui sont fournis par les sujets ou les institutions provinciales (requête, avis, projets). Le concept d'ordonnances promulguées motu proprio paraît ainsi plus qu'évanescent au regard du processus d'élaboration de la législation. Ceci nous amène à questionner le travail des conseils centraux dans les Pays-Bas espagnols. Le cas étudié a permis de mettre en avant les Archiducs, l'audiencier (Verreycken) mais également un officier provincial au champ d'action plus vaste (Peckius). D’autres conseillers ont-ils été impliqués dans la préparation du texte de mars 1618 ou bien Peckius a-t-il joui d'une liberté d'action ? De manière générale, il faut retenir que ce n'est pas parce qu'un travail est mené collectivement au sein d'un conseil de gouvernement que toutes les décisions sont prises ensemble. En d'autres mots, collectivité n'est pas synonyme de collégialité.

On serait peut-être amené à s'interroger sur l'imposition unilatérale ou la négociation des dispositions législatives en raison des échanges effectués entre gouvernés et gouvernants tout au long du processus décisionnel. Il faut d'ailleurs souligner que Madrid n'intervient pas dans le processus législatif au cours du règne des Archiducs89. Si d'aucuns ont pu mettre en avant qu'une décision unilatérale au niveau législatif ne reflète pas la réalité de l'époque, il serait trop radical de parler dans le cas présent d'un droit négocié. La requête de 1618 illustre parfaitement la capacité 
Chacun pour soi(e) ? Lobbying, stratégies économiques et commerce internation...

des sujets à émettre des observations ou soumettre des propositions. Les Pays-Bas habsbourgeois ne sont pas encore au $\mathrm{XVI}^{\mathrm{e}}$ et au début du $\mathrm{XVII}^{\mathrm{e}}$ siècle un ensemble territorial uniforme qui répond comme un seul homme aux injonctions et décisions du pouvoir central. On peut sans doute parler d'un droit imposé si on s'accorde à reconnaître au prince et ses représentants la capacité à prendre la décision ultime. Mais il s'agit néanmoins d'une décision pesée à la balance des informations disponibles. Dès lors, l'imposition d'un droit prescrit s'avère toujours plus évidente au cours du $\mathrm{XVI}^{\mathrm{e}}$ siècle induisant un ressac de l'activité législative des juridictions inférieures $\underline{90}$. Il y a néanmoins comme un jeu d'aller-retour entre les niveaux juridictionnels et agents sociaux qui autorise à ne pas voir l'émergence de structures centralisées comme la résultante de la seule décision du souveraing1.

\section{Notes}

1 Archives générales du Royaume (Bruxelles, cité ci-après AGR), Papiers d'État et de l'Audience (cité ci-après PEA), $\mathrm{n}^{\circ} 114$, non folioté et non daté (repris avec les documents préparatoires à l'ordonnance sur les soies du 3 mars 1618).

2 BRANTS V., La Belgique au XVII ${ }^{e}$ siècle. Albert et Isabelle. Études d'histoire politique et sociale, Louvain-Paris, 1910 ; Thomas W. et Duerloo L., Albert \& Isabella, 1598-1621. Essays, Turnhout, 1998 ; MARTYN G., Het Eeuwig Edict van 12 juli 1611. Zijn genese en zijn rol in de verschriftelijking van het privaatrecht, Bruxelles, 2000 ;VAN WYHE C. (dir.), Isabel Clara Eugenia. Female Sovereignty in the Courts of Madrid and Brussels, Londres, 2011 ; DuERLOo L., Dynasty and Piety. Archduke Albert (1598-1621) and Habsburg Political Culture in an Age of Religious Wars, Aldershot, 2012.

$\underline{3}$ Bulst N., Zum Problem städtischer und territorialer Kleider-, Aufwands- und Luxusgesetzgebung in Deutschland (13. - Mitte 16. Jahrhundert), dans Renaissance du pouvoir législatif et genèse de l'état, (dir.) A. Gouron et A. RigaudièRe, Montpellier, 1988, p. 20-57 ; ID., Les ordonnances somptuaires en Allemagne : expression de l'ordre social urbain (XIV ${ }^{e}-X V I^{e}$ siècle), dans Comptes rendus des séances de l'Académie des Inscriptions et Belles-Lettres, t. 137, 1993, p. 771-783 ; ID., Kleidung als sozialer Konfliktstoff : Probleme kleidergesetzlicher Normierung im sozialen Gefüge, dans Jahrbuch für Universalgeschichte, t. 44, 1993, p. 32-46 ; FOGEL M., Modèle d'État et modèle social de dépense : les lois somptuaires en France de 1485 à 1660, dans Genèse de l'État moderne. Prélèvement et redistribution. Actes du colloque de Fontevraud 1984, (dir.) J.-Ph. GenET et M. Le MenÉ, Paris, 1987, p. 227-235 ; Kovesi Killerby C., Sumptuary Law in Italy 1200-1500, Oxford, 2002 ; VAN PETEGHEM P., Les édits somptuaires à travers l'histoire : réalités et fictions, dans Langage et droit à travers l'histoire. Réalités et fictions, (dir.) G. van Dievoet, Ph. Godding, D. van den AuweEle, Louvain-Paris, 1989, p. 227-248.

4 D'aucuns ont argumenté qu'il convenait même d'abandonner le terme « État » au risque d'une réification outrancière et d'en faire une entité autonome ne se laissant jamais saisir par la recherche. Cf. ABrams Ph., Notes on the Difficulty of Studying the State (1977), dans Journal of Historical Sociology, t. 1, 1988, p. 58-89 ; Bourdieu P., Sur l'État. Cours au Collège de France (1989-1992), Paris, 2012, p. 157.

5 REINHARD W., No Statebuilding from Below! A Critical Commentary, dans Empowering Interactions. Political Culture and the Emergence of the State in Europe 1300-1900, (dir.) W. 
Blockmans, A. Holenstein, J. Mathieu, avec la collaboration de D. SchäPpi, Aldershot, 2009, p. 299-304.

6 Vermeesch G., Professional Lobbying in Eighteenth-century Brussels : The Role of Agents in Petitioning the Central Government Institutions in the Habsburg Netherlands, dans Journal of Early Modern History, t. 16, 2012, p. 96.

7 En contexte colonial, on dénote d'importantes différences dans les modalités de communication en matière juridique entre les Couronnes espagnole et anglaise, par exemple. Cf. Ross R. J., Legal Communications and Imperial Governance : British North America and Spanish America Compared, dans The Cambridge History of Law in America, (dir.) M. GRossBerg et Ch. TomLINS, t. 1, Cambridge, 2008, p. 104-143.

8 HAEMERS J., Ad petitionem burgensium. Petitions and peaceful resistance of craftsmen in Flanders and Mechelen (13th-16th centuries), dans Los grupos populares en la ciudad medieval europea, (dir.) J. A. Solórzano Telechea, B. Arízaga Bolumburu et J. HAEmers, Logroño, 2014, p. 377-378.

$\underline{9}$ Pour un aperçu succinct à l'automne du moyen âge voir LowAGIE H., The Political Functions of Oral Networks in the Later Medieval Low Countries, dans The Voices of the People in Late Medieval Europe. Communication and Popular Politics, (dir.) J. Dumolyn, J. HaEmers, H. R. Oliva HERRER et V. CHALLET, Turnhout, 2014, p. 205-213.

10 Pour le $\mathrm{XV}^{\mathrm{e}}$ siècle et le début du siècle suivant, nous renvoyons aux travaux suivants : HAEMERS J., Ad petitionem burgensium..., op. cit., p. 371-394 ; ID., Geletterd verzet. Diplomatiek, politiek en herinneringscultuur van opstandelingen in de laatmiddeleeuwse en vroegmoderne stad (casus : Gent en Brugge), dans Bulletin de la commission royale d'histoire, t. 176, 2010, $\mathrm{n}^{\circ} 1$, p. 5-54.

11 C'est ce que tend à montrer Jonas Braekevelt à propos de la législation de Philippe le Bon pour le comté de Flandre (1419-1467). Nos propres recherches nous ont amené à confirmer, pour le $\mathrm{XVI}^{\mathrm{e}}$ siècle, les propos émis par Jonas Braekevelt. Et ce non seulement en matière législative mais aussi dans le domaine administratif (requête pour disposer d'un office, par exemple). Cf. BraekeVelt J., Popular Voices within Princely Legislation : Assessing the Discourse of Flemish Petitions and Burgundian Narrationes, dans The Voices of the People in Late Medieval Europe..., op. cit., p. 149-165. Voir exemples : AGR, Conseil privé espagnol (cité ci-après CPE), n 194 (premier registre des actes de A. de Laloo, secrétaire du Consejo supremo de Flandes y Borgoña, janvier 1588-décembre 1589).

12 Simon N., Un dossier "gracieusement" conservé. La genèse de l'ordonnance d'octobre 1541 sur le droit de grâce, dans Bulletin de la commission royale pour la publication des anciennes lois et ordonnances de Belgique, t. 53, 2012, p. 131-132.

13 Rospocher M., Beyond the Public Sphere : A Historiographical Transition, dans Beyond the Public Sphere. Opinions, Publics, Spaces in Early Modern Europe, (dir.) M. RospocheR, BologneBerlin, 2012, p. 22.

14 Pour une critique des thèses de J. Habermas et la démonstration que le concept d'opinion publique peut être valablement défendu pour les anciens Pays-Bas, on consultera : BLOEMENDAL J. 
Chacun pour soi(e) ? Lobbying, stratégies économiques et commerce internation...

et van Dixhoorn A., Literary Cultures and Public Opinion in the Early Modern Low Countries, dans Literary Cultures and Public Opinion in the Early Modern Low Countries, (dir.) J. Bloemendal et A. van DixHOORN, Leiden, 2011, p. 1-36.

15 Il est évidemment impossible de déterminer la proportion de requêtes conservées ou même celle des requêtes qui ont effectivement été satisfaites. Cf. CAUCHIES J.-M., La législation princière dans le comté de Hainaut : ducs de Bourgogne et premiers Habsbourg (1427-1506). Contribution à l'étude des rapports entre gouvernants et gouvernés dans les Pays-Bas à l'aube des temps modernes, Bruxelles, 1982, p. 92.

16 Constatation établie sur la base du dépouillement des séries de minutes préparatoires aux ordonnances conservées par le gouvernement central des Pays-Bas espagnols pour la période 1580-1610. Cf. AGR, PEA, n 1103-1112, n²1145-1147.

17 M. Van Gelder l'a bien montré concernant les marchands originaires des Pays-Bas espagnols et des Provinces-Unies actifs à Venise (les deux sont assimilés aux marchands « flamands »). Entre 1581 et 1651, ce sont plus d'une centaine de requêtes (collectives et individuelles) qui sont transmises par ces marchands aux autorités vénitiennes. Nous renvoyons également à la contribution de D. De Ruysscher et J. Puttevils dans ce numéro. Cf. VAN GELDER M., Trading Places. The Netherlandish Merchants in Early Modern Venice, Leiden-Boston, 2009, p. 139-152.

18 Sur la Trêve de Douze Ans voir LeSAFFer R. (dir.), The Twelve Years Truce (1609) Peace, Truce, War and Law in the Low Countries at the Turn of the 17th Century, Leiden, 2014 ; EsTEBAN ESTRíngana A., La Tregua de los Doce Años : fracaso del principo de reunión pactada de los Países Bajos bajo el dominio de los Archiduques, dans Pedralbes, t. 29, 2009, p. 95-157.

19 Pierre Peckius, chancelier de Brabant, écrit à l'archiduchesse Isabelle en mars 1621 que les États généraux des Provinces-Unies étaient particulièrement étonnés d'entendre « ladite proposition en ce qui concernoit le point de rejoindre toutes les parties et membres des Pays-Bas en un corps et soubz un chef, et d'entrer en accord soubz la recognoissance des Princes naturelz et sur le pied ancien tel qu'il estoit devant les troubles (...) ». Les États généraux tenaient « pour maxime indubitable et infaillible que lesdits Estatz ont acquis pleine et entiere liberté, comme elle a esté declairée devant le traicté de la Trefve et par la teneur d'icelluy et que lesditz Estatz s'y arrestent en telle sorte qu'ilz ne peuvent ouyr parler de chose quelconque tendant directement ou indirectement au contraire (...) ». Cf. AGR, PEA, n 1377 (lettre de Pierre Peckius à Isabelle, 03/ 03/1621, en partie chiffrée).

20 HERRERo SÁnchez M., La monarchie espagnole et le capital marchand. Les limites de la guerre économique et la lutte pour la suprématie dans l'espace atlantique, dans Guerre et économie dans l'espace atlantique du XVI $I^{e}$ au XX $X^{e}$ siècle, (dir.) S. MARZAGALli et B. MARNOT, Bordeaux, 2006, p. 201 ; ID., Republican Monarchies, Patrimonial Republics. The Catholic Monarchy and the Mercantile Republics of Genoa and the United Provinces, dans Polycentric Monarchies. How did Early Modern Spain and Portugal Achieve and Maintain a Global Hegemony ?, (dir.) P. CARDIM, T. HERZOG, J. J. RuIZ IBÁÑEZ, G. SABATINI, Eastbourne, 2012, p. 181-196.

21 Eliott J., Imperial Spain, 1469-1716, Londres, 2002, p. 321 ; HeRzog T., Frontiers of Possession. Spain and Portugal in Europe and the Americas, Cambridge (Ma)-Londres, 2015, p. 7.

$\underline{22}$ Anvers demandait déjà vers 1603 que soit accordé « le libre commerce avec ceux de 
Hollande ». Cf. PRIMS F., Geschiedenis van Antwerpen, t. VI, nouvelle édition illustrée ( $1^{\mathrm{e}}$ édition : 1929-1931), Bruxelles, 1982, p. 364 ; LÓPEZ MARTíN I., Embargo and protectionist policies. Early Modern Hispano-Dutch relations in the Western Mediterranean, dans Mediterranean Studies, t. 7, 1998, p. 191-215.

$\underline{23}$ De 1595 à 1795, on estime à un million le nombre d'individus ayant effectué un voyage jusqu'en Asie à partir des Provinces-Unies. Cf. BROOK T., Le chapeau de Vermeer. Le XVII e siècle à l'aube de la mondialisation, Paris, 2012, p. 34.

24 Braudel F., Civilisation matérielle, économie et capitalisme (XV $V^{e}$-XVIII $I^{e}$ siècles), t. 2, Paris, 1979, p. 79.

25 VAN Gelder M., Trading Places..., op. cit., p. 6-9.

26 PUTTEVILs J., Klein gewin brengt rijkdom in. De Zuid-Nederlandse handelaars in de export naar Italië in de jaren 1540, dans Tijdschrift voor sociale en economische geschiedenis, t. 6, 2009, p. 26-52.

27 VAN Gelder M., Trading Places..., op. cit., p. 58 ; PUTTEVILS J., 'Eating the bread out of their mouth' : Antwerp's export trade and generalized institutions, 1544-5, dans The Economic History Review, 2015, DOI : 10.1111/ehr.12103 (accessible en ligne mais non inséré dans un numéro au moment de la consultation le 24 juin 2015).

28 VAN GELDER M., Trading Places..., op. cit., p. 41.

29 Anvers attire l'attention des gouvernants en raison du capital financier disponible compte tenu des échanges qui y sont menés entre marchands européens. Toutefois, si la ville brabançonne fut un locus of capital accumulation (Ch. Tilly), la pression fiscale des gouvernants, constamment à la recherche de fonds, ne permit pas à Anvers de devenir une bargaining metropole (W. Blockmans) de premier ordre. Cf. DE VIJLDER N., Voor de vorst, voor vrijheid en voor recht. Een comparatieve analyse van het stedelijke fiscale en financiële beleid in de zeventiende eeuw, dans Tijdschrift voor sociale en economische geschiedenis, t. 9, 2012, p. 50-52. Pour un bilan historiographique : Vermeesch G., De haas en de schilpad. Stad en staat in pre-industrieel Europa, dans Stadsgeschiedenis, t. 5, 2010, p. 56-71.

30 Blockmans W. et 't HaRt M., Power, dans The Oxford Handbook of Cities in World History, (dir.) P. ClaRK, Oxford, 2013, p. 423.

31 Pour le Florentin, l'hégémonie économique mondiale anversoise durant une partie du XVI siècle découlait de sa position dominante au sein de l'économie européenne. C'est le lieu où arrivent les biens du Portugal, de l'Espagne, de la France, de l'Italie, de l'Angleterre, etc., c'est-àdire d'entités politiques qui disposaient pour la plupart de colonies asiatiques ou américaines. Guichardin soulignait encore l'importance de Venise dans les échanges commerciaux liés aux produits de luxe et de la soie. Cf. VAN Dixhoorn A., The Values of Antwerp and the prosperity of Belgica. Political economy in Guicciardini's Descrittione di tutti i Paesi Bassi (1567), dans Trading Values in Early Modern Antwerp, (dir.) Ch. GöTTLER, B. RAMAKERS et J. WOOdALL, Leiden-Boston, 2014, p. 77-106.

32 Anvers demanda fermement, au moment des négociations préparatoires à la Trêve, que le 
Chacun pour soi(e) ? Lobbying, stratégies économiques et commerce internation...

trafic maritime via l'Escaut soit rapidement rétabli. Cf. PRIMS F., Geschiedenis van Antwerpen..., op. cit., p. 145.

33 PutteVils J., Klein gewin..., op. cit., p. 42 ; Thijs A., De Zijdenijverheid te Antwerpen in de zeventiende eeuw, Bruxelles, 1969, p. 4.

34 VAN GeLDER M., Trading Places..., op. cit., p. 76.

35 Les Anglais quittèrent toutefois la ville à la suite de la furie espagnole de 1576. Cf. PRIMS F., Geschiedenis van Antwerpen..., op. cit., p. 25-26.

36 PutteVils J., 'Eating the bread out of their mouth'..., op. cit. ; BlondÉ B. et VAN DAMme I., Early Modern Europe : 1500-1800, dans The Oxford Handbook of Cities in World History..., op. cit., p. 246.

37 LuU L., Immigrants and the diffusion of skills in Early Modern London : the case of silk weaving, dans Documents pour l'histoire des techniques, t. 15, 2008, p. 34.

38 La requête de 1618 indique bien que « semblablement, les passementiers lesquelz sont en très grand nombre en ces pays (dependants aussi de la manufacture des soyes) en seroient aussi grandement préjudiciéz par la révocation dudict placcart » du 3 mars de la même année. Cf. AGR, PEA, $\mathrm{n}^{\circ} 1114$, non folioté (article 15 de la requête).

39 LUU L., Immigrants and the diffusion of skills..., op. cit., p. 35.

40 Puttevils J., Trading Silks and Tapestries in Sixteenth-Century Antwerp, dans Europe's Rich Fabric. The Consumption, Commercialisation, and Production of Luxury Textiles in Italy, the Low Countries and Neighbouring Territories (14th-16th centuries), (dir.) B. LAMBERT et K. WILSON, Aldershot, p. 131-158.

41 AGR, PEA, n 1114, non folioté (article 9 de la requête).

42 Pour rappel, le terme «flamand » englobe, pour les Vénitiens, les marchands originaires des Pays-Bas espagnols et des Provinces-Unies. Cf. note 17 supra.

$4 \underline{3}$ Venise imposait d'emprunter des routes traversant son hinterland afin de garantir une activité économique en certains endroits de la Terraferma. Cf.VAN GELDER M., Trading Places..., op. cit., p. 93-95.

44 On se limitera à citer ici : DE MuncK B., Lourens P. et LuCASSEN J., The establishment and distribution of craft guilds in the Low Countries 1100-1800, dans Craft Guilds in the Early Modern Low Countries. Work, Power and Representation, (dir.) M. PRAK, C. LIS, J. LuCASSEN et H. Soly, Aldershot, 2006, p. 32-73 ; DE Munck B., La qualité du corporatisme. Stratégies économiques et symboliques des corporations anversoises, $\mathrm{XVI}^{e}$-XVIII ${ }^{e}$ siècles, dans Revue d'histoire moderne et contemporaine, t. 54, 2007, p. 116-144 ; ID., Gilding golden ages : perspectives from Early Modern Antwerp on the guild debate, c. 1450-c. 1650, dans European Review of Economic History, t. 15, 2011, p. 221-253.

45 Recueil des ordonnances des Pays-Bas. Règne d'Albert et Isabelle (cité ci-après ROPB. Albert et Isabelle), (éd.) V. BranTs, t. 2, Bruxelles, 1912, p. 410. 
46 THijs A., De Zijdenijverheid..., op. cit., p. 2.

47 ROPB. Albert et Isabelle, t. 1, p. 302-303.

48 La requête est envoyée par le Magistrat de la ville en collaboration avec les « doyens, anciens et supposts de la nation de la mercercie » de la ville. Cf. Archives de l’État à Anderlecht, Conseil de Brabant, $n^{\circ} 5192, f^{\circ} 112 r$.

49 La requête indique qu'il « sera trouvé estrange que ceulx qui sont en troubles et confusion surpassent ces pays par bonnes, louables et politicques ordonnances ». Dès lors, « voz altesses serenissimes soyent servies d'y pourveoir par une ordonnance generale a publier par tout le pays de leur obeissance et en cas de besoing en prennant l'advis des aultres provinces et consaulx ». Cf. Ibid., f ${ }^{\circ} 113 \mathrm{v}$.

$\underline{50}$ On sait d'ailleurs que le Magistrat lillois fut bien informé de cette requête dès le 14 septembre 1605. Cf. Ibid., f¹13v-114r ; THIJ A., De Zijdenijverheid..., op. cit., p. 25.

51 ROPB. Albert et Isabelle, t. 2, p. 411.

52 ROPB. Albert et Isabelle, t. 1, p. 302.

$\underline{53}$ Le mûrier est nécessaire à la culture de la soie car il permet le développement des chenilles responsables de la production des fils de soie.

54 Brants V., La Belgique au XVII ${ }^{e}$ siècle..., op. cit., p. 186.

$\underline{55}$ L'octroi est conféré à Thomas Gramaye le 6 mars 1607. Cf. ROPB. Albert et Isabelle, t. 1, p. 323.

$\underline{56}$ THijs A., De Zijdenijverheid..., op. cit., p. 1.

57 Cf. Van Peteghem P., Les édits somptuaires..., op. cit., passim.

58 PutTeVils J., Trading Silks and Tapestries..., op. cit. ; BlondÉ B. et VAN DAMme I., Early Modern Europe..., op. cit., p. 254.

59 Recueil des ordonnances des Pays-Bas, $2^{\text {e }}$ série, t. 3, (éd.) J. LAMEERE, Bruxelles, 1902, p. 271-272 ; CAuchies J.-M., “Oyez et faites paix...”, De Baudouin de Constantinople à Joseph II : six siècles de législation aux anciens Pays-Bas, dans Académie royale de Belgique. Bulletin de la Classe des Lettres, $6^{\mathrm{e}}$ série, t. 14, 2003, p. 300.

60 L'ordonnance aborde d'autres thématiques que celle du luxe des vêtements (crimes en état d'ivresse, usure, etc.). Cf. ROPB, t. 5, (éd.) J. LAMEERE et H. SimONT, Bruxelles, 1910, p. 214-215.

61 ROPB, t. 6, (éd.) J. LAMEERE, Bruxelles, 1922, p. 80-83. Voir la portée pour la ville d'Anvers : PRIMS F., Geschiedenis van Antwerpen..., op. cit., p. 280 ; VAN CAUWENBERGS Cl., L'industrie de la soie à Anvers depuis 1532 jusqu'à nos jours, dans Bulletin de la Société royale de géographie d'Anvers, t. 12, 1887, p. 111.

62 AGR, PEA, n²02, n. f. (lettre de l'archiduc Ernest, gouverneur général des Pays-Bas, à Philippe II, 28/05/1594). 
Chacun pour soi(e) ? Lobbying, stratégies économiques et commerce internation...

63 Le Conseil n'indique pas avec précision les actes auxquels il fait référence. Voir Archives de l’État Anderlecht, Conseil de Brabant, n 4885, f282v (avis du Conseil de Brabant à l'archiduc Ernest, gouverneur général des Pays-Bas, 20/09/1594 (minute)).

64 Né à Louvain en 1562, Pierre Peckius occupa plusieurs postes importants au sein des institutions centrales et provinciales des Pays-Bas espagnols : conseiller au Grand Conseil de Malines (1601), surintendant de la justice militaire (1610), conseiller au Conseil privé (lettres patentes du 24 octobre 1610), d'abord nommé adjoint au chancelier de Brabant (1614) il devient chancelier en 1616. Cf. THOMAs C., De l'affection, avec laquele je me dispose de la servir toute ma vie. Prosopographie des grands commis du gouvernement central des Pays-Bas espagnols (1598-1700), t. 2, Bruxelles, 2011, p. 572-573.

65 Né dans les années 1550, il est nommé en juillet 1578 au poste d'audiencier à la suite de son beau-frère, Pierre d'Overloope. Il resta en poste jusqu'à son décès en 1621. Il effectua de nombreuses missions pour le compte des Archiducs : négociations préparatoires à la Paix de Vervins entre l'Espagne et la France (1598) ou celles tenues à La Haye avant la signature de la Trêve de Douze Ans (1607). Cf. Ibid., p. 737-738 ; LEFĖVRE J., Les Verreycken. Audienciers et premiers secrétaires d'État au gouvernement des Pays-Bas, dans L'Intermédiaire, t. 45, 1953, p. 401-408.

66 P. Peckius indique que «son Alteze m'a commandé de vous envoyer la minute cy jointe (...)». Cf. AGR, PEA, n 1114, non folioté (lettre du 11/02/1618 conservée avec les documents relatifs à l'ordonnance du 03/03/1618).

67 Le premier paragraphe de la requête signale que celle-ci est envoyée par les « preparateurs de soyes de la ville d'Anvers joinct aveq eulx ceulx du meisme exercice des villes de Bruges, Denremonde, Malines, Liere et aultres » (c'est nous qui soulignons). Cf. AGR, PEA, n 1114, non folioté [Lorsque nous mentionnons les articles de la requête, nous nous sommes permis de ne pas indiquer systématiquement la cote d'inventaire du fonds des Papiers d'État et de l'Audience par souci d'ergonomie de lecture].

$\underline{68}$ Article 5 de la requête.

69 Pour asseoir les rapports qui ont longtemps lié Anvers et l'Italie, le Magistrat anversois met en avant, à maintes reprises, les échanges commerciaux entre ces deux régions européennes. Limiter les exportations ou réguler drastiquement l'autorisation de commercer avec des territoires étrangers constitueraient des dispositions fatales à l'économie de la cité brabançonne. Cf. PRIMS F., Geschiedenis van Antwerpen..., op. cit., p. 361.

70 Article 3 de la requête.

71 Article 10 de la requête.

$\underline{72}$ Article 18 de la requête.

73 Article 19 de la requête.

74 Article 21 de la requête.

75 C'est notamment le cas avec les travailleurs du comté de Namur. En août 1598, l'archiduc 
Albert interdit aux batteurs de cuivre de partir travailler en Lorraine. Dans les années 1620, le Conseil provincial contacte l'archiduchesse Isabelle en raison des forgerons tentés de partir en Suède. Cf. AEN, États provinciaux, n $742-743$ (ordre d'Albert à la ville de Namur (8 août 1598), copie) - Brants V., La Belgique au XVII e siècle..., op. cit., p. 180 ; Simon N, Les Archiducs, la guerre et la religion : facteurs d'influences sur la décision politique dans les Pays-Bas espagnols (ca.1620-ca.1635), dans Religion et piété au défi de la guerre de Trente Ans, (dir.) Ph. MARTIN et B. FORCLAZ, Rennes, 2015, p. 44-46.

76 Article 6 de la requête.

77 Article 11 de la requête.

78 Article 13 de la requête.

79 Article 17 de la requête.

80 Articles 14, 15 et 16 de la requête.

81 En soulignant que les problèmes procèdent « notoirement par la connivence et peu de debvoirs que noz officiers rendent a l'entretenement dudict placcart », le gouvernement central reprend un poncif présent dans de nombreux textes législatifs. En effet, les officiers apparaissent comme les seuls et uniques responsables dans la mesure où l'on ne peut alors concevoir que le prince puisse lui-même mal agir. Cf. ROPB Albert et Isabelle, t. 2, p. 424-425.

82 HAEMERS J., Ad petitionem burgensium..., op. cit., p. 385.

$\underline{83}$ C'est le cas notamment dans la principauté ecclésiastique de Bamberg à la fin du XVI ${ }^{\mathrm{e}}$ siècle où les potiers de certaines localités réalisent un lobbying auprès du prince-évêque afin de favoriser leur activité dans la principauté. Des tensions survinrent après la promulgation de l'acte quand les métiers de la poterie non consultés se sentent trahis. Cf. STAUdenMaIER J., Participation and protest. Actors and Interests in the Early Modern Legislation Process. A Case Study from the Prince-Bishopric of Bamberg, dans Rebellion and resistance, (dir.) H. JENSEN, Pise, 2009, p. 51-68.

84 Certains marchands anversois spécialisés dans la soie ou la tapisserie informent, au cours des années 1540-1550, leurs clients italiens dès qu'ils ont vent de projets législatifs. Cf. PUTTEVILS J., Trading Silks and Tapestries..., op. cit.

85 L'acte est publié entre octobre et novembre 1597 dans les Pays-Bas. La lettre envoyée au Conseil de Brabant est datée du 6 octobre, tandis que celle transmise au Conseil provincial de Namur indique le 8 novembre. L'ordonnance de Rodolphe II est quant à elle datée du $1^{\mathrm{e}}$ août 1597. Cf. AGR, PEA, n 1109, non folioté (06/10/1597) - Archives de l’État à Namur (AEN), Conseil provincial, $\mathrm{n}^{\circ} 185, \mathrm{f}^{\circ} 254 \mathrm{r}-263 \mathrm{r}$.

86 Article 26 de la requête des métiers de la soie.

87 Il en va ainsi des multiples interventions de l'Italien Giovanni Battista Ferrufini auprès du gouvernement central de Bruxelles sans qu'il prenne la peine d'informer le Magistrat anversois de son projet d'ordonnance sur les assurances maritimes. Voir la contribution de D. De Ruysscher et J. Puttevils dans ce numéro. 
Chacun pour soi(e) ? Lobbying, stratégies économiques et commerce internation...

88 Vermeesch G., Professional Lobbying in Eighteenth-century Brussels..., op. cit.

89 Martyn G., How 'Sovereign' were the Southern Netherlands under the Archdukes ?, dans The Twelve Years Truce (1609)..., op. cit., p. 201.

90 De SChepper H., Die Einheit der Niederlande unter Karl V. - Mythos oder Wirklichkeit ?, dans Karl V. 1500-1558. Neue Perspektiven seiner Herrschaft in Europa und Übersee, (dir.) A. KoHLER, B. HAIDER et Ch. OtTNER, avec la collaboration de M. Fuchs, Vienne, 2002, p. 475-487.

91 HolENSTEIN A., Introduction : Empowering Interactions : Looking at Stabuilding from below, dans Empowering Interactions. Political Culture and the Emergence of the State in Europe 1300-1900, (dir.) W. Blockmans, A. Holenstein, J. MathieU, avec la collaboration de D. SchäPPI, Aldershot, 2009, p. 1-31.

PDF généré automatiquement le 2020-07-08 08:22:09

Url de l'article : https://popups.uliege.be:443/1370-2262/index.php?id=327 